\title{
Research on residual useful life prediction of li-ion battery based on GA-LM-BPNN
}

\author{
Yanyi $\mathrm{Xu}^{1}$, Jianqiao $\mathrm{Xu}^{2}$ \\ Department of Information Security, Naval University of Engineering, Wuhan, 430033, China \\ ${ }^{2}$ Corresponding author \\ E-mail: ${ }^{1} 569121080 @ q q . c o m,{ }^{2} x u j i a n q i a o 321 @ 163 . c o m$
}

Received 28 October 2021; received in revised form 22 November 2021; accepted 29 November 2021 DOI https://doi.org/10.21595/vp.2021.22273

Check for updates

Copyright (C) 2021 Yanyi Xu, et al. This is an open access article distributed under the Creative Commons Attribution License, which permits unrestricted use, distribution, and reproduction in any medium, provided the original work is properly cited.

\begin{abstract}
Prediction of the residual useful life of Lithium-ion batteries is one of the hotspots presently. In order to further obtain the residual useful life prediction of Li-ion battery, the degeneration data of it obtained from university of Maryland are analyzed. Discrete data point filtering is performed on the degraded data to simplify the processing. Due to the defects of slow learning speed and easy to fall into local minimum of the Back Proragation Neural Network (BPNN), the fast speed of Levemberg Marquardt (LM) algorithm and the globally search advantage of Genetic Algorithm (GA) are used to deal with. The construction of GA-LM-BPNN is three layers and is used to predict the residual capacity of $\mathrm{Li}$-ion batteries.
\end{abstract}

Keywords: Residual useful life prediction, Li-ion Battery, GA-LM-BPNN.

\section{Introduction}

The prediction of Li-ion battery is the focus of the whole residual useful life (RUL) prediction [1]. Dong proposed a sub-optimal method for health diagnosis of satellite Li-ion batteries based on Auto Vector Regression and Particle Filter (AVR-PF) [2]. In order to overcome the problems of high dependence model and poor adaptability of single prediction method, Back Proragation Neural Network (BPNN) was improved, and the fusion data-driven method was adopted to predict the RUL of Li-ion battery [3].

\section{Test conduct and analysis of li-ion battery}

The charging and discharging process of lithium-ion batteries is the same as the process of completing the corresponding energy conversion. In the production process of $\mathrm{Li}$-ion batteries, irreversible chemical reactions occur inside the batteries, resulting in the electrode loss and increasing internal resistance [4-5]. These changes in internal resistance are the main parameters corresponding to the degradation. An important step is to establish its life degradation model. However, it is not easy to establish the ideal model of battery degradation accurately in practical application. Based on a large number of experimental data, NASA's PCoE research center proposed a simple and feasible battery equivalent model to predict the RUL. The equivalent model is shown in Fig. 1.

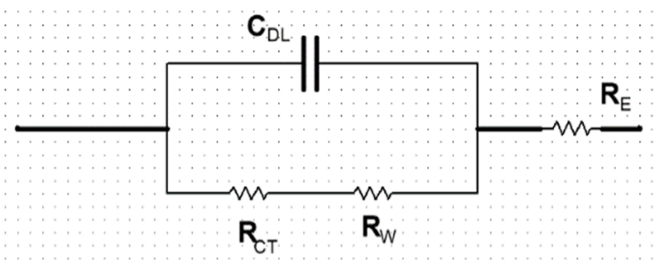

Fig. 1. Equivalent model

Where, $C_{D L}$ is the double-layer capacitance; $R_{C T}$ is the transfer resistance; $R_{W}$ is the Warburg impedance, and $R_{E}$ is the electrolyte resistance. After analyzing the data, NASA researchers found 
that the effect of $R_{W}$ and $C_{D L}$ on the life degradation is negligible, but has a high linear relationship with $R_{C T}+R_{E}$. They verified a high linear correlation between the capacity and impedance parameters of Li-ion batteries, as shown in Fig. 2. Saha et al. proposed the following empirical model to describe Li-ion batteries [6]:

$C_{k+1}=\eta_{C} C_{k}+\beta_{1} \exp \left(-\frac{\beta_{2}}{\Delta t_{k}}\right)$

where, $C_{k}$ is the capacity in the $k$ cycles; $\Delta t_{k}$ is the rest time between two adjacent charging and discharging cycles; $\beta_{1}$ and $\beta_{2}$ is a specific parameter of the battery empirical model. $\eta_{C}$ is the coulomb efficiency, which is used to express the percentage between the total amount of charge released by the battery and the total amount of charge charged. In Li-ion batteries, increasing the rest time after charging and discharging can increase the capacity in the next cycles.

It is not difficult to see from the above analysis that the capacity will gradually decrease with the aging of Li-ion battery. The capacity charging is basically gradually away from the nominal capacity, so it can be used to predict the RUL of capacity.

In this paper, the test data of capacity to be used is $1.1 \mathrm{Ah}$. The whole experiment data is mainly composed of the discharge of the charging part and the battery capacity. The charging experimental data includes impedance data, charging voltage and charging current data. The discharge experimental data includes impedance data, discharge voltage and discharge current data, as well as the corresponding ambient temperature test time and other information. The experimental process can be described as follows.

(1) The ambient temperature of the experiment is $20^{\circ} \mathrm{C}-25^{\circ} \mathrm{C}$;

(2) Charging mode: the constant current is $0.675 \mathrm{~A}$ and the maximum voltage is $4.2 \mathrm{~V}$;

(3) Discharge mode: the constant current is $1.35 \mathrm{~A}$, the minimum voltage is $2.7 \mathrm{~V}$, and the discharge rate is $1 \mathrm{C}$;

(4) Electrochemical impedance spectroscopy was used for measurement;

(5) Select the Capacity-CS2-8\#, Capacity-CS2-33\#, Capacity-CS2-34\#of Battery Data Set as the experimental data. The degradation curve is shown in Fig. 3. When the battery capacity reaches $80 \%$ of the nominal capacity, the experiment will be stopped. Because the nominal capacity is $1.1 \mathrm{Ah}$, and the capacity threshold is $0.88 \mathrm{Ah}$.

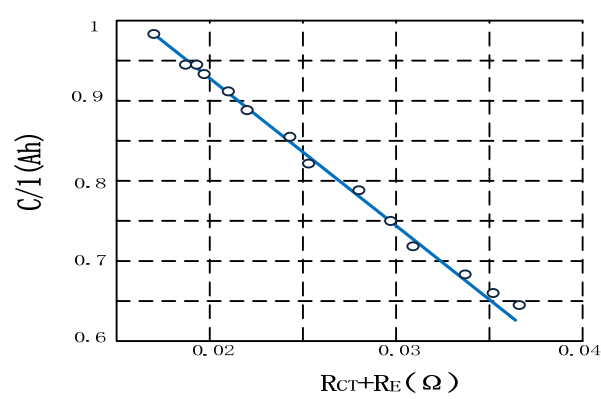

Fig. 2. Linear correlation between capacity and impedance

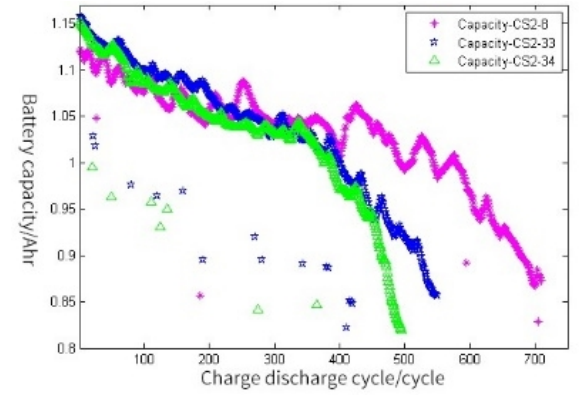

Fig. 3. Degradation curve of Li-ion battery capacity

It can be seen from the above experimental data, at the beginning of the prediction, the number of historical data is limited, so it is still strict with the predictive modeling method and demanding because of interference or man-made wrong operation in the experiment, as well as some abnormal data in the data set. Thus, it is necessary to carry out the raw data preprocessing on the abnormal data points firstly. Since the number of charge and discharge cycles of lithium-ion batteries has reached 500 to 700 , and many corresponding test data points can reduce the calculation efficiency. Thus, the degradation trend of Li-ion battery can obtain 8 data. For example, simplify the original 
data and return one data reduction data every 5 data intervals. The degradation trend of is consistent with the original data obviously. In order to evaluate the prediction accuracy, the definition is as follows:

$R U L_{\text {Error }}=\left|R U L_{\text {Actualvalue }}-R U L_{\text {Predictivevalue }}\right|$,

where, $R U L_{\text {Actualvalue }}$ is the true RUL of the Li-ion battery, and $R U L_{\text {Predictivevalue }}$ is the RUL value predicted by the model method.

\section{GA optimize LM-BPNN modeling}

\subsection{Implementation of LM Algorithm}

LM algorithm is a fast algorithm that combines the advantages of Gauss-Newton method and gradient descent method, which has the advantages of fast convergence and global search. The vector formed by the weights of the $k$ iteration is $\omega^{(k)}$, so that:

$\omega^{(k+1)}=\omega^{(k)}+\Delta \omega$.

Input error index function of neural network $E(\omega)$ is the following:

$E(\omega)=\frac{1}{2} \sum_{k=1}^{N}\left(d_{k}-y_{k}\right)^{2}=\frac{1}{2} \sum_{k=1}^{N} e_{k}^{2}(\omega)$.

The adjustment rule obtained by solving the error function of BP neural network by least square method is as follows:

$\Delta(\omega)=-\left[\nabla^{2} E(\omega)\right]^{-1} \nabla(\omega)$.

The modified Gauss-Newton method is that:

$\Delta(\omega)=-\left[J^{T}(n) J(n)\right]^{-1} J(n) e(n)$.

LM algorithm can improve Gauss-Newton method as follows:

$\Delta(\omega)=-\left[J^{T}(n) J(n)+\mu I\right]^{-1} J(n) e(n)$.

Among them, $I$ is identity matrix, $\mu$ is the proportional coefficient, and is greater than zero. When it is close to a solution value, the weight adjustment is like Gauss-Newton method. When it is far from a solution value, the weight adjustment is like gradient descent method. Its descent speed is much faster than that of the gradient method, and it is proved by experiments that the descent speed is several tens to one hundred times higher than that of the gradient method, which greatly improves the performance of the neural network.

\subsection{GA optimize Im-bpnn modeling}

The weights and thresholds parameters of BPNN are generally random Numbers between $[-0.5,0.5]$, but these parameters have a great impact on the training effect of the $\mathrm{NN}$, and cannot be accurately obtained. To solve this problem, GA optimization is adopted in this paper to get the best initial weights and thresholds. GA is based on the selection principle of survival of the fittest in nature. It is a global optimization search artificial intelligence algorithm with strong robustness. GA-optimized LM-BP network is mainly divided into three parts: the determination of network topology, the initial weight and threshold of GA optimization, and the prediction of network after 
weight and threshold updating.

\section{Prediction of li-ion battery based on GA-LM-BPNN}

\subsection{Determine the topology of BPNN}

According to Kolmgorov theorem, the structure of BPNN is three-layer, which can meet all the accuracy requirements to achieve the numerical approximation of continuous function. All of them will obviously affect the battery capacity. Take them all as input variables of NN in order to predict battery capacity as the output variable of BPNN. The number of neurons in the hidden layer is determined by the golden optimization method. The number of nodes in the input layer and output layer of BPNN is this, so the value range of the number of nodes in the hidden layer is calculated:

$a=\frac{m+i}{2}=\frac{5+1}{2}=3 \leq i \leq(m+i+10)=(5+1+10)=16=b$.

According to the above analysis, the 5-6-1 three-layer structure of BPNN is determined. Using this BPNN structure, there are 42 weights and thresholds, and the individual coding length of subsequent GA is 42 , as shown in Table 1 . Then dividual is encoded by real numbers.

Table 1. Weights and threshold counter

\begin{tabular}{|c|c|}
\hline Weights and thresholds of topological layers & Bits \\
\hline From input layer to hidden layer & 30 \\
\hline Hidden layer & 6 \\
\hline From hidden layer to output layer & 6 \\
\hline Output layer & 1 \\
\hline
\end{tabular}

\subsection{Research on BPNN prediction}

Carry out 100 debugging for the parameters of three-layer BP network, and determine that the number of iterations is set as 100 , the learning rate is set as 0.1 , and the training target is set as $0.004 \%$. The test data are filtered, and the noise signal is removed and normalized. 2000 groups of data are randomly selected, 1900 groups are used as training data, and 100 groups are used as test data. Then, the test set is input into the BPNN model to predict the output, and the result is shown in Fig. 4. For the selected test data set, the error is shown in Fig. 4.

It is not difficult to see from Fig. 4 that the overall output result of BPNN is quite good, but there is still some prediction error, mainly concentrated in the initial stage of prediction. The error is still a little large, and the maximum reaches 0.0297 .

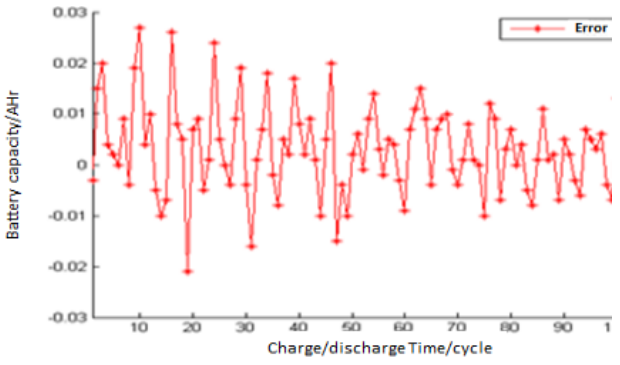

Fig. 4. Predict error of BPNN

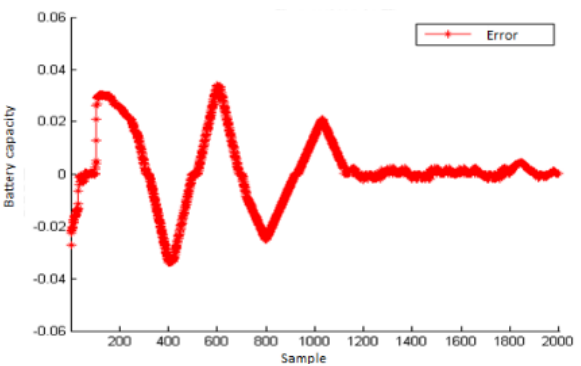

Fig. 5. Predict error of BPNN samples

It is not difficult to see from Fig. 5 that the overall prediction effect is good when training samples are learned according to the structure of BPNN. The maximum error is 0.03 . After the training samples reach 1200, the prediction error becomes smaller and smaller, which is relatively 
stable with little fluctuation.

\subsection{Prediction of LM-BPNN by GA optimization}

Firstly, the structure of BPNN is 5-6-1. Then code length of the GA individual is 42 bits; the evolutionary algebra of GA is 50 , and the population size is set as 30 . The crossover operation sets conducted by the roulette method. The crossover probability is set as 0.8 ; the mutation probability is 0.01 , and the fitness error function is $F$. According to the steps of GA-LM-BPNN, the curve of fitness function changes in the GA optimization process decreases with the increase of algebra. It indicates that the prediction error is getting smaller and smaller, and the optimal weights and thresholds obtained after 50 generations, which are shown in Table 2.

Table 2. Optimal weights and threshold

\begin{tabular}{|l|c|c|c|c|c|c|}
\hline \multirow{4}{*}{$\omega_{\text {mi }}$} & 1.4819 & 1.4931 & -2.7276 & -2.2447 & -0.9881 & -1.9257 \\
\cline { 2 - 7 } & -0.9496 & -2.5244 & 1.8420 & -2.5901 & -0.0168 & -2.0345 \\
\cline { 2 - 7 } & 1.0456 & -0.5914 & -2.0804 & -0.0187 & 0.9711 & 1.5852 \\
\cline { 2 - 7 } & -1.3385 & -0.3675 & 1.0724 & 1.1690 & 0.9329 & -1.7788 \\
\cline { 2 - 7 } & -2.7230 & -0.7106 & 1.5464 & 1.5931 & -1.9729 & 1.8532 \\
\hline $\mathrm{a}_{\mathrm{i}}$ & 1.7532 & 1.7712 & 1.4588 & -0.3265 & -0.0877 & 0.2911 \\
\hline$\omega_{\text {ip }}$ & 2.8814 & -1.1865 & 0.6284 & -2.1562 & -0.4892 & 1.3088 \\
\hline $\mathrm{b}_{\mathrm{p}}$ & -0.6668 & - & - & - & - & - \\
\hline
\end{tabular}



Fig. 6. Predict result of GA-LM-BP network

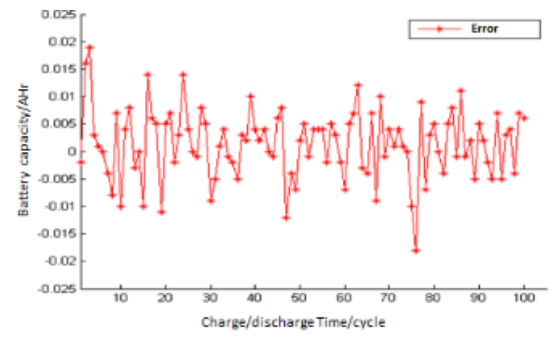

Fig. 7. Predict error of GA-LM-BP network

The optimal weights and thresholds are assigned to BPNN, and the optimized network is used to train. Then the output of the whole system is predicted, as shown in Fig. 6, Fig. 7.

It is not difficult to see from Fig. 6 and Fig. 7 that GA-LM-BPNN is more capable than BPNN in fitting nonlinear functions, with significantly reduced prediction errors and improved accuracy. From Fig. 8 and Fig. 9, GA-LM-BPNN has better output prediction effect, with the highest prediction error of 0.0195 and the average error of 0.0045 . By comparing the prediction output effect of BPNN and GA-LM-BPNN, the prediction result is more accurate and the stability is improved after the initial weight and threshold value are optimized by GA. The prediction error is significantly improved and the global convergence is good.

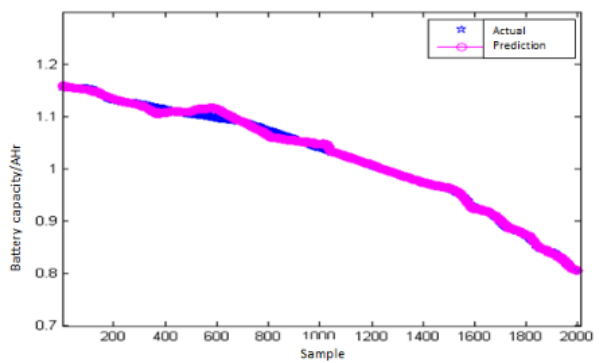

Fig. 8. Predict output of GA-LM-BP network

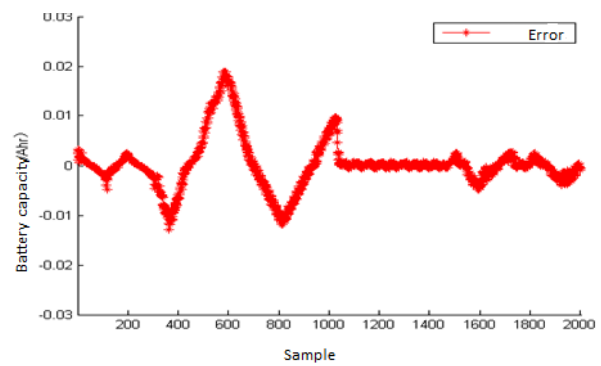

Fig. 9. Predict error of GA-LM-BP network 


\section{Conclusions}

The LM algorithm and GA algorithm are introduced into BP neural network algorithm. It not only combines the rapidity of gauss-Newton method and gradient descent method, but also optimizes the initial weight and threshold of BP neural network to reduce the probability of falling into the local minimum. Fuel by increasing computer power and algorithmic advances, machine learning techniques have become powerful tools for finding patterns of data. The field of quantum machine learning explores how to devise and implement quantum software that could enable machine learning faster than that of classical computers. The application in the prediction of Lithium-ion batteries will have a huge effect.

\section{Acknowledgements}

This work is supported by the National Defense Preresearch Foundation 9140A27020113JB11393 and 9140A27020314JB11438.

\section{References}

[1] Z. S. Zhang, "Research on modeling and fault prediction of Li-ion batteries," Master's Thesis, Dalian Maritime University, 2016.

[2] H. C. Dong, "Research on diagnosis method of satellite power battery health," Ph.D. Thesis, Harbin Institute of Technology, 2015.

[3] Y. Miao, P. Hynan, A. Von Jouanne, and A. Yokochi, "Current Li-ion battery technologies in electric vehicles and opportunities for advancements," Energies, Vol. 12, No. 6, p. 1074, Mar. 2019, https://doi.org/10.3390/en12061074

[4] W. Li, K. R. Crompton, C. Hacker, and J. K. Ostanek, "Comparison of current interrupt device and vent design for 18650 format lithium-ion battery caps," Journal of Energy Storage, Vol. 32, p. 101890, Dec. 2020, https://doi.org/10.1016/j.est.2020.101890

[5] G. Yang and W. Yu, "Eddy current damper capable of collecting electric energy," Vibroengineering PROCEDIA, Vol. 33, pp. 28-33, Oct. 2020, https://doi.org/10.21595/vp.2020.21672

[6] B. Saha, K. Goebel, and J. Christophersen, "Comparison of prognostic algorithms for estimating remaining useful life of batteries," Transactions of the Institute of Measurement and Control, Vol. 31, No. 3-4, pp. 293-308, Jun. 2009, https://doi.org/10.1177/0142331208092030 